\title{
RBPMS is differentially expressed in high-grade serous ovarian cancers.
}

Shahan Mamoor ${ }^{1}$

1shahanmamoor@gmail.com

East Islip, NY 11730

Ovarian cancer is the most lethal gynecologic cancer ${ }^{1}$. We sought to identify genes associated with high-grade serous ovarian cancer (HGSC) by comparing global gene expression profiles of normal ovary with that of primary tumors from women diagnosed with HGSC using published microarray data ${ }^{2,3}$. We found significant differential expression of RBPMS in high-grade serous ovarian tumors.

Keywords: ovarian cancer, high-grade serous ovarian cancer, HGSC, targeted therapeutics in ovarian cancer, systems biology of ovarian cancer, RBPMS.

PAGE 1 OF 13 
The five-year survival rate for women diagnosed with high-grade serous ovarian cancer is between $30-40 \%$ and has not changed significantly in decades 4,5 . The development of novel, targeted therapeutics to treat HGSC can be facilitated by an enhanced understanding of the transcriptional behavior of ovarian tumors relative to that of the normal ovary. We mined published microarray data ${ }^{2,3}$ to compare global gene expression profiles between HGSC ovarian tumors and that of normal ovarian tissue. We identified the gene encoding RBPMS as among the most differentially expressed genes in HGSC tumors of the ovary. RBPMS may be a gene of interest when prioritizing the study of target genes and pathways for the development of novel therapeutic interventions in high-grade serous ovarian cancers.

\section{Methods}

We used microarray data from datasets and GSE $124766^{2}$ and GSE $146556^{3}$ for this differential gene expression analysis of high-grade serous ovarian carcinomas. The Benjamini and Hochberg method of p-value adjustment was used for ranking of differential expression but raw p-values were used for assessment of statistical significance of global differential expression. Log-transformation of data was auto-detected, and the NCBI generated category of platform annotation was used. GSE124766 was generated using Agilent-014850 Whole Human Genome Microarray $4 \mathrm{x} 44 \mathrm{~K}$ G4112F with $\mathrm{n}=3$ of for normal ovarian tissue and $\mathrm{n}=8$ for tumors from patients with high-grade serous ovarian cancer. GSE146556 was generated using Affymetrix 
Human Gene 1.0 ST Array with $n=3$ for normal ovarian tissue and $n=40$ for tumors from patients with high-grade serous ovarian cancer. GEO2R provides mRNA expression levels only for the top 250 most differentially expressed genes.

A statistical test was performed to evaluate whether RBPMS expression was significantly different when comparing normal ovarian tissue from control subjects and primary tumors from women diagnosed with HGSC using a two-tailed, unpaired t-test with Welch's correction. Only p-values less than 0.05 were considered statistically significant. We used PRISM for all statistical analyses (Version 8.4.0)(455).

\section{Results}

We mined published microarray data ${ }^{2,3}$ to identify differentially expressed genes in highgrade serous ovarian cancer (HGSC), the type of ovarian cancer responsible for $70-80 \%$ of deaths resulting from the most lethal gynecologic malignancy.

RBPMS is differentially expressed in ovarian tumors from women diagnosed with HGSC.

We identified the gene encoding RBPMS as among the genes whose expression was most different when comparing primary HGSC tumors to normal ovarian tissue ${ }^{2}$ (Table 1). We found that RBPMS was differentially expressed in primary HGSC tumors (Table 1). When sorting all of the transcripts measured by microarray based on change in expression between HGSC and the normal ovary, RBPMS ranked 132 out of 41093 total transcripts (Table 1). Differential

PAGE 3 OF 13 
expression of RBPMS in HGSC tumors was statistically significant (Table 1; $\mathrm{p}=5.05 \mathrm{E}-06$ ).

We analyzed a second microarray dataset ${ }^{3}$ generated using normal ovarian tissues and tumors from women diagnosed with HGSC to determine whether differential expression of RBPMS could be observed in tumors from a separate group of patients. We again found that RBPMS was differentially expressed in primary HGSC tumors (Table 2). When sorting all of the transcripts measured by microarray based on change in expression between HGSC and the normal ovary, in this data set, RBPMS ranked 2212 out of 29088 total transcripts (Table 2). Differential expression of RBPMS in HGSC tumors in this second microarray dataset was statistically significant (Table $2 ; \mathrm{p}=1.23 \mathrm{E}-03)$.

RBPMS is expressed at lower levels in HGSC when compared to the normal ovary.

We obtained exact mRNA expression levels for the differentially expressed RBPMS transcript from both normal ovarian tissue and from high-grade serous ovarian tumors. RBPMS was expressed at lower levels in high-grade serous ovarian cancers when compared to the normal ovary; this difference was statistically significant (Figure $1 ; \mathrm{p}=0.0026$ ). We calculated a mean fold change of $0.7338 \pm 0.0466$ in RBPMS expression when comparing HGSC tumors to normal ovarian tissue (Table 1).

Thus, we found using published microarray data that RBPMS was among the genes most differentially expressed in tumors from women with high-grade serous carcinomas and that RBPMS expression was lower in HGSC tumors when compared to the normal ovary. 


\section{Discussion}

Transcriptional profiling of HGSC in a Cancer Genome Atlas integrated genomic analysis classified HGSC into four subtypes based on gene clustering: immunoreactive, differentiated, proliferative, and mesenchymal ${ }^{6}$. We sought to continue to describe the transcriptional landscape of high-grade serous ovarian cancers ${ }^{6}$ and identify genes whose differential expression was associated with HGSC by using published microarray data from primary tumors of women diagnosed with HGSC compared to transcriptome data from normal ovarian tissue ${ }^{2,3}$. In both datasets analyzed, RBPMS was among the genes whose expression changed most significantly when comparing the normal ovary to primary HGSC tumors.

RBPMS, or RNA-binding protein with multiple splicing, is an RNA-binding protein and the human homologue of Xenopus Hermes ${ }^{7}$. RBPMS is one member of a large family of RRM (RNA Recognition Motif) proteins that possess one RRM motif. Highest expression of RBPMS is in the developing heart ${ }^{8}$. In the vegetal cortex of the Xenopus oocyte, Hermes is degraded within hours during oocyte maturation, and depletion of Hermes results in cleavage defects of vegetal blastomeres ${ }^{9}$. In stage I oocytes, Hermes interacts with nanos and this interaction requires RNA localization signals in the nanos-3'-UTR. hnRNP I also interacts with Hermes and may be a co-factor required for the interaction between nanos and Hermes within germinal granules $^{10}$. In the central nervous system, RBPMS has exclusive expression in retinal ganglion cells, and an established function of RBPMS is in the regulation of axon arbor formation in RGC11. Deficiency of Hermes protein in vivo leads to defects in axon arbor complexity in the 
optic tectum in both Xenopus and zebrafish and defects in granule localization ${ }^{11}$.

In differentiated vascular smooth muscle cells, RBPMS is a regulator of mRNA splicing. RBPMS expression is down-regulated during switching of SMCs from contractile to motile phenotypes; $20 \%$ of the alternative splicing (AS) changes during this phenotypic switch could be attributed to RBPMS ${ }^{12}$. RBPMS regulated alternative splicing of cytoskeletal elements including actin and focal adhesion machinery, as well as splicing of the SMC transcription factor Myocardin, suggesting RBPMS is a master regulator of AS in SMCs ${ }^{12}$.

Investigators used PAR-CLIP (photo-activatable-ribonucleoside-enhanced cross linking and immunoprecipitation) in HEK293 cells to identify RNA interaction partners of RBPMS and found that RBPMS bound tandem CAC motifs separated by spacers variable in length; in vitro, RBPMS could bind $(\mathrm{AC})_{9}$ and $(\mathrm{CAC})_{6}$ motifs $^{13}$. Specific RNA messages bound by RBPMS in PAR-CLIP included NDUFA6, UBE2V1, SRM and ETF1'13. In the free state, RBPMS forms a symmetrical dimer, and mutations in the dimerization and RNA-binding interface of RRM motif result in disruption of RBPMS dimerization and decreased RNA-binding affinity ${ }^{14}$.

Multiple studies implicate RBPMS in some aspect of ovarian cancer pathogenesis and in the biology of other human cancers. RBPMS is over-expressed in the A2780 cervical cancer cell line and depletion of RBPMS in A2780 cells using small interfering RNA (siRNA) resulted in an increase in invasive potential, while over-expression of RBPMS in cisplatin-resistant A2780CP20 cells resulted in decreases in cell proliferation ${ }^{15}$. A study identifying differentially expressed genes between recurrent and non-recurrent ovarian cancer found that RBPMS was 
among the most differentially expressed genes (DEGs) between these groups and formed a node when generating a protein-protein interaction network based on interaction between DEGs (16). miR-375 was a differentially expressed microRNA in this analysis, and RBPMS was identified as a target gene of miR-37516. Investigators studying miR-21-3p in ovarian cancer found that depletion of miR-21-3p resulted in decreases in proliferation and invasion in vitro; luciferase assays identified RBPMS as a target of miR-21-3p ${ }^{17}$. In cell culture, depletion of RBMPS reduced sensitivity to cisplatin treatment ${ }^{17}$. In a group of Korean patients with stage IIIC HGSC, a one of 27 genes located in a region of copy number loss on 8p21.1-p1218. In drug-resistant multiple myeloma (MM) cells, polycomb group protein EZH2 was identified as over-expressed, and associated with worse prognosis in MM patients; using chromatin immunoprecipitation of the H3K27me3 mark, investigators identified RBPMS as a target of EZH219. miR-138 was identified as an EZH2-targeting microRNA, and over-expression of EZH2, like depletion of RBPMS, resulted in higher resistance of MM cells to anti-myeloma therapies ${ }^{19}$. miR-138 overexpression restored RBPMS and restored sensitivity to MM chemotherapeutics in vitro ${ }^{19}$. RBPMS has also been described as up-regulated in dedifferentiated hepatocellular carcinomas (HCC) and in HCC with loss of $13 q^{20}$.

Differential expression of RBPMS in HGSC should be validated in larger and separate cohorts of women with HGSC. If validated, mouse models of high-grade serous ovarian cancers $^{21-23}$ can be utilized to assess the effect of conditional expression of RBPMS in HGSC tumor growth post-development and on survival in vivo. 


\section{References}

1. Guppy, A.E., Nathan, P.D. and Rustin, G.J., 2005. Epithelial ovarian cancer: a review of current management. Clinical Oncology, 17(6), pp.399-411.

2. Zhang, W., Klinkebiel, D., Barger, C.J., Pandey, S., Guda, C., Miller, A., Akers, S.N., Odunsi, K. and Karpf, A.R., 2020. Global DNA hypomethylation in epithelial ovarian cancer: passive demethylation and association with genomic instability. Cancers, 12(3), p.764.

3. Hoffmann, K., Berger, H., Kulbe, H., Thillainadarasan, S., Mollenkopf, H.J., Zemojtel, T., Taube, E., Darb-Esfahani, S., Mangler, M., Sehouli, J. and Chekerov, R., 2020. Stable expansion of high-grade serous ovarian cancer organoids requires a low-Wnt environment. The EMBO journal, 39(6), p.e104013.

4. Bowtell, D.D., Böhm, S., Ahmed, A.A., Aspuria, P.J., Bast Jr, R.C., Beral, V., Berek, J.S., Birrer, M.J., Blagden, S., Bookman, M.A. and Brenton, J.D., 2015. Rethinking ovarian cancer II: reducing mortality from high-grade serous ovarian cancer. Nature reviews Cancer, 15(11), pp.668-679.

5. Vaughan, S., Coward, J.I., Bast, R.C., Berchuck, A., Berek, J.S., Brenton, J.D., Coukos, G., Crum, C.C., Drapkin, R., Etemadmoghadam, D. and Friedlander, M., 2011. Rethinking ovarian cancer: recommendations for improving outcomes. Nature Reviews Cancer, 11(10), pp.719-725.

6. Cancer Genome Atlas Research Network, 2011. Integrated genomic analyses of ovarian carcinoma. Nature, 474(7353), p.609.

7. Shimamoto, A., Kitao, S., Ichikawa, K., Suzuki, N., Yamabe, Y., Imamura, O., Tokutake, Y., Satoh, M., Matsumoto, T., Kuromitsu, J. and Kataoka, H., 1996. A unique human gene that spans over $230 \mathrm{~kb}$ in the human chromosome 8p11-12 and codes multiple family proteins sharing RNA-binding motifs. Proceedings of the National Academy of Sciences, 93(20), pp. 10913-10917.

8. Gerber, W.V., Yatskievych, T.A., Antin, P.B., Correia, K.M., Conlon, R.A. and Krieg, P.A., 1999. The RNA-binding protein gene, hermes, is expressed at high levels in the developing heart. Mechanisms of development, 80(1), pp.77-86.

9. Zearfoss, N.R., Chan, A.P., Wu, C.F., Kloc, M. and Etkin, L.D., 2004. Hermes is a localized factor regulating cleavage of vegetal blastomeres in Xenopus laevis. Developmental biology, 267(1), pp.60-71.

10.Aguero, T., Zhou, Y., Kloc, M., Chang, P., Houliston, E. and King, M.L., 2016. Hermes (Rbpms) is a critical component of RNP complexes that sequester germline RNAs during oogenesis. Journal of developmental biology, 4(1), p.2.

PAGE 8 OF 13 
11.Hörnberg, H., Wollerton-van Horck, F., Maurus, D., Zwart, M., Svoboda, H., Harris, W.A. and Holt, C.E., 2013. RNA-binding protein Hermes/RBPMS inversely affects synapse density and axon arbor formation in retinal ganglion cells in vivo. Journal of Neuroscience, 33(25), pp. 10384-10395.

12.Nakagaki-Silva, E.E., Gooding, C., Llorian, M., Jacob, A.G., Richards, F., Buckroyd, A., Sinha, S. and Smith, C.W., 2019. Identification of RBPMS as a mammalian smooth muscle master splicing regulator via proximity of its gene with super-enhancers. Elife, 8, p.e46327.

13.Farazi, T.A., Leonhardt, C.S., Mukherjee, N., Mihailovic, A., Li, S., Max, K.E., Meyer, C., Yamaji, M., Cekan, P., Jacobs, N.C. and Gerstberger, S., 2014. Identification of the RNA recognition element of the RBPMS family of RNA-binding proteins and their transcriptomewide mRNA targets. Rna, 20(7), pp.1090-1102.

14.Teplova, M., Farazi, T.A., Tuschl, T. and Patel, D.J., 2016. Structural basis underlying CAC RNA recognition by the RRM domain of dimeric RNA-binding protein RBPMS. Quarterly reviews of biophysics, 49 .

15.Báez-Vega, P.M., Valiyeva, F., Santiago, G. and Vivas-Mejia, P., 2017. The role of RBPMS in cisplatin resistant ovarian cancer.

16.Wang, X., Han, L., Zhou, L., Wang, L. and Zhang, L.M., 2018. Prediction of candidate RNA signatures for recurrent ovarian cancer prognosis by the construction of an integrated competing endogenous RNA network. Oncology reports, 40(5), pp.2659-2673.

17.Báez-Vega, P.M., Vargas, I.M.E., Valiyeva, F., Encarnación-Rosado, J., Roman, A., Flores, J., Marcos-Martínez, M.J. and Vivas-Mejía, P.E., 2016. Targeting miR-21-3p inhibits proliferation and invasion of ovarian cancer cells. Oncotarget, 7(24), p.36321.

18.Kwon, J.Y., Seo, Y.R. and Ahn, W.S., 2011. Recognition of potential predictive markers for diagnosis in Korean serous ovarian cancer patients at stage IIIc using array comparative genomic hybridization with high resolution. Molecular \& Cellular Toxicology, 7(1), pp.77-86.

19.Rastgoo, N., Pourabdollah, M., Abdi, J., Reece, D. and Chang, H., 2018. Dysregulation of EZH2/miR-138 axis contributes to drug resistance in multiple myeloma by downregulating RBPMS. Leukemia, 32(11), pp.2471-2482.

20.Drozdov, I., Bornschein, J., Wex, T., Valeyev, N.V., Tsoka, S. and Malfertheiner, P., 2012. Functional and topological properties in hepatocellular carcinoma transcriptome. PloS one, 7(4), p.e35510.

21.Harrington, B.S., He, Y., Davies, C.M., Wallace, S.J., Adams, M.N., Beaven, E.A., Roche, D.K., Kennedy, C., Chetty, N.P., Crandon, A.J. and Flatley, C., 2016. Cell line and patient- 
derived xenograft models reveal elevated CDCP1 as a target in high-grade serous ovarian cancer. British journal of cancer, 114(4), pp.417-426.

22.Perets, R., Wyant, G.A., Muto, K.W., Bijron, J.G., Poole, B.B., Chin, K.T., Chen, J.Y.H., Ohman, A.W., Stepule, C.D., Kwak, S. and Karst, A.M., 2013. Transformation of the fallopian tube secretory epithelium leads to high-grade serous ovarian cancer in Brca; Tp53; Pten models. Cancer cell, 24(6), pp.751-765.

23.Kim, J., Coffey, D.M., Creighton, C.J., Yu, Z., Hawkins, S.M. and Matzuk, M.M., 2012. High- grade serous ovarian cancer arises from fallopian tube in a mouse model. Proceedings of the National Academy of Sciences, 109(10), pp.3921-3926. 
1

2

\section{Table 1: RBPMS is differentially expressed in high-grade serous ovarian carcinomas.}

The rank of differential expression, the probe/transcript ID, the p-value with respect to global differential expression, $t$, a moderated $t$-statistic, $B$, the log-odds of differential expression between the two groups compared, the fold change of RBPMS in HGSC tumors when compared to the normal ovary, the gene and gene name are listed in this chart. 


\begin{tabular}{|c|l|l|l|l|l|l}
\hline Rank & ID & p-value & t & B & Gene & Gene name \\
\hline 2212 & 8145669 & $1.23 E-03$ & -3.4529684 & -1.2615066 & RBPMS & $\begin{array}{l}\text { RNA binding protein } \\
\text { with multiple splicing }\end{array}$ \\
\hline
\end{tabular}

Table 2: RBPMS is differentially expressed in high-grade serous ovarian carcinomas.

The rank of differential expression, the probe/transcript ID, the p-value with respect to global differential expression, $\mathrm{t}$, a moderated $\mathrm{t}$-statistic, $\mathrm{B}$, the log-odds of differential expression between the two groups compared, the fold change of RBPMS in HGSC tumors when compared to the normal ovary, the gene and gene name are listed in this chart.

PAGE 12 OF 13 


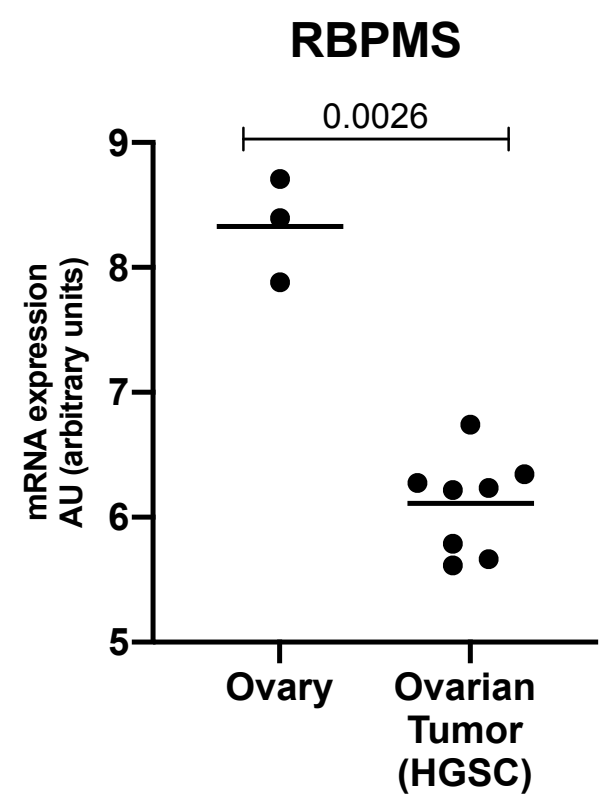

Figure 1: RBPMS is expressed at significantly lower levels in HGSC tumors when compared to the normal ovary.

The mRNA expression of RBPMS in normal ovarian tissues from control subjects (left) and in the primary tumors of patients with HGSC (right) is represented with mean mRNA expression level marked and the result of a statistical test evaluating the significance of difference in RBPMS expression between the normal ovary and primary tumors from patients with HGSC, a p-value, listed above. 